\title{
Freqüência, precisão e localização temporal de conhecimento de resultados e o processo adaptativo na aquisição de uma habilida- de motora de controle da força manual
}

\author{
Go Tani \\ Cássio M. Meira Jr. \\ Fábio R. F. Gomes
}

https://doi.org/10.5628/rpcd.05.01.59

\section{RESUMO}

No processo de aquisição de habilidades motoras, importa tanto a estabilização funcional, como a capacidade de aplicação do que foi adquirido a novas situações, caracterizando um processo cíclico e dinâmico de instabilidade-estabilidade-instabilidade, que resulta em crescente complexidade. O presente estudo investigou a variável conhecimento de resultados (CR) no processo adaptativo em aprendizagem motora. De acordo com os resultados, regimes de CR menos freqüentes, menos precisos e com atividade no intervalo pós-CR (fatores de instabilidade, incerteza e desordem) não prejudicaram o processo adaptativo de aquisição de uma habilidade motora de controle de força manual.

Palavras-chave: conhecimento de resultados (CR), controle de força, processo adaptativo, aquisição de habilidades motoras, aprendizagem motora.

\author{
Universidade de São Paulo \\ Escola de Educação Física e Esporte \\ Brasil
}

\begin{abstract}
Frequency, precision and temporal localization of knowledge of results and the adaptive process in the acquisition of a manual force control skill.

In motor skill acquisition, not only functional stability is important, but also the capability of applying what was acquired to new contexts; this characterises a cyclic and dynamic process of instability-stability-instability, which results in increasing complexity. This study aimed to investigate the role of knowledge of results (KR) in motor learning viewed as an adaptive process. According to the results, KR regimes of low frequency and precision, and with interpolated activity on post-KR interval (factors of instability, uncertainty and disorder) were not prejudicial to adaptive process in acquiring a manual force control motor task.
\end{abstract}

Key Words: knowledge of results (KR), feedback, force control, adaptive process, motor skill acquisition, motor learning. 


\section{INTRODUÇÃO}

"A prática cujos resultados são conhecidos leva à perfeição" é a alternativa apresentada por Bartlett (4) à máxima "a prática leva à perfeição". A proposição de Bartlett expressa de forma mais completa o processo de aquisição de habilidades motoras porque estabelece uma vinculação entre prática e conhecimento de resultados (CR) - duas variáveis cruciais de aprendizagem.

A associação desses dois fatores pode ser melhor compreendida mediante uma apreciação do processo pelo qual um indivíduo aprende uma habilidade motora. Em primeiro lugar, o objetivo de performance é estabelecido. O objetivo é geralmente a solução de um problema motor que surge normalmente no meio ambiente externo. Definido o objetivo, o indivíduo procura desenvolver a melhor maneira de alcançá-lo, ou seja, processa informações, seleciona um plano de ação que atenda apropriadamente às demandas do momento e, finalmente, executa o movimento. Durante a execução, o indivíduo recebe informações sobre como está sendo executado o movimento e após a sua conclusão recebe informações que o permitem avaliar se o movimento executado alcançou ou não o objetivo almejado. Essas informações são denominadas genericamente de feedback.

Normalmente, as primeiras tentativas de execução resultam em grandes erros de performance. $\mathrm{O}$ indivíduo toma consciência desses erros mediante feedback e, com base no seu processamento, decide sobre qual mudança introduzir na próxima tentativa para que o objetivo seja alcançado. Em outras palavras, o mecanismo de detecção e correção de erro é acionado. Como resultado, um novo plano de ação é elaborado, executado, avaliado, e esse processo é repetido até a obtenção do objetivo ou a solução adequada do problema motor.

Durante esse processo, é muito comum o indivíduo tomar consciência do erro cometido, mas não ser capaz de detectar a sua origem e, conseqüentemente, ser incapaz de corrigi-lo. As informações que ele obtém do próprio sistema sensorial sobre a execução do movimento e o seu resultado no meio ambiente são denominadas feedback intrínseco ou inerente. Numa situação de incapacidade para detectar a causa dos erros, o indivíduo necessita receber informações adicionais de fontes externas sobre a execução e o resultado do movimento. Essas informações, denominadas feedback extrínseco ou aumentado, são normalmente fornecidas pelo professor, pesquisador, técnico ou sistema de videoteipe. O feedback extrínseco é freqüentemente denominado conhecimento de resultados (CR) e o seu efeito na aprendizagem motora tem sido intensamente estudado desde o começo do século XX.

Os estudos pioneiros sobre CR em aprendizagem motora remontam a um dos experimentos clássicos de Thorndike (51) sobre a lei do efeito. Essa lei, em linhas gerais, estabelece relação entre a natureza da resposta e a avaliação posterior que lhe está associada, e portanto, o CR constitui-se num fator vital para ocorrência de aprendizagem. O experimento em questão constou de habilidades motoras em que os sujeitos, com os olhos vendados, desenhavam linhas de diferentes tamanhos. O CR foi fornecido na forma "errado" e "certo" (quando dentro de uma faixa de tolerância). O resultados mostraram que o grupo que praticou com CR evoluiu de uma porcentagem de respostas corretas de $13 \%$ no pré-teste para 55\% ao final da fase de aquisição. Já o grupo sem CR não obteve melhoras ao longo das tentativas. Trowbridge e Cason (52) corroboraram as conclusões de Thorndike e foram além, propondo que regimes de CR mais precisos (em valores quantitativos) produziam melhor aprendizagem comparados a regimes de CR geral.

Esse foi o panorama conceitual e experimental de onde se originaram os estudos acerca do CR, mostrando que quando ele é apresentado após uma resposta, melhora o desempenho. Essa melhora esteve relacionada a dois fatores: associativo e motivacional. A interpretação do papel associativo do CR remete a questões sobre a natureza do processo de aprendizagem, uma vez que ele funcionaria como um guia que orienta em direção a solução do problema. A função motivacional do CR esteve relacionada a interesses militares e industriais com o propósito de aumentar o desempenho individual por meio do alcance de metas, posto que o CR, atuando como fonte de energização, estimularia o indivíduo a consolidar as respostas que já estariam incorporadas no seu repertório (2).

Na Psicologia Experimental, havia uma crença de que o CR teria o mesmo efeito na aprendizagem 
humana que o reforço positivo tinha na aprendizagem animal. Isso acontecia em virtude de os pesquisadores acreditarem em leis gerais que poderiam ser aplicadas de maneira equivalente a animais e humanos. Bilodeau e Bilodeau (7) manipularam CR em 25,33 e $100 \%$ das tentativas na aprendizagem de posicionamento de uma alavanca e não detectaram diferenças significativas na aquisição. Por outro lado, o mesmo não aconteceu com reforço positivo em animais. Por exemplo, Boren (8) propôs que a taxa de pressionamento da barra que fornecia alimento para ratos em vários regimes aumentou na medida em que a proporção de reforços diminuiu. $\mathrm{O}$ atraso de $\mathrm{CR}$, correspondente ao atraso no fornecimento de reforço em animais, foi a variável com maior incongruência de resultados. Skinner (45) afirmou, baseado na premissa de que o reforço imediato é fundamental em animais, que o CR deveria ser imediato, mas as pesquisas não deram suporte a essa afirmação $(7,22,30)$. Pesquisas sobre o intervalo de atraso de CR foram o prenúncio do papel informacional do $\mathrm{CR}$ em aprendizagem motora, além do associativo e motivacional.

Com a proposição da teoria de circuito fechado (1) e da teoria de esquema (41), nas quais o CR desempenha papel fundamental para a aprendizagem de movimentos, a sua função foi ligada à formação de estruturas cognitivas (traços e esquemas, respectivamente). Com forte influência dessas duas teorias, os principais aspectos do CR que receberam inicialmente maior atenção dos pesquisadores foram: a) freqüência de $\mathrm{CR}$; b) precisão de $\mathrm{CR}$; c) localização temporal de CR; d) retirada de CR; e e) CR errôneo. Os resultados desses estudos mostraram, no seu conjunto, que quanto mais freqüente, mais preciso, mais imediato e mais rico do ponto de vista informacional, maior o seu efeito na aprendizagem.

Entretanto, essa posição sobre os princípios de CR foi contestada por Salmoni, Schmidt e Walter (39), os quais, ao reexaminarem a metodologia empregada nos estudos até então realizados, detectaram um problema que colocava dificuldades na generalização dos resultados: a maioria dos estudos não separava claramente os efeitos temporários de performance dos efeitos mais duradouros de aprendizagem em virtude da ausência de um delineamento de transferência. Segundo esses autores, quando administrado nas tentativas de aquisição, o CR desempenha função orientadora (o aprendiz confia nas propriedades de informação contidas no CR que o guia para responder na próxima tentativa) e motivadora (os indivíduos supostamente são indiferentes quando ele não aparece e se esforçam bastante quando ele está presente). Quando o CR desempenha essas funções é difícil detectar o que contribui para a aprendizagem. Tentativas de teste sem CR, portanto, são importantes para avaliar a aprendizagem, haja vista os resultados de Boulter (9) com atividades interpoladas no intervalo entre tentativas.

Existia, portanto, a necessidade de um reapreciação sobre o efeito dessa variável na aquisição de habilidades motoras. De fato, o que ocorreu a partir da "denúncia" de Salmoni et al. (39) foi uma avalanche de estudos reexaminando os fatores clássicos de CR como sua precisão $(15,33,40)$, freqüência relativa $(16,17,31,44,57,53)$ e acumulação $(23,43,59$, 57). Além disso, os estudos sobre o efeito de atividades interpoladas ao CR foram retomados $(3,6,29$, 47), assim como novos fatores começaram a ser investigados, dentre os quais os tipos de CR, especialmente sobre a cinemática do movimento ( 37 , 42), o efeito de CR na aquisição de programas motores generalizados e sua parametrização $(56,58)$, a influência de CR errôneo $(10,11,35)$, a faixa de tolerância de erro $(13,24,44,46)$ e o CR auto-controlado $(14,17,27,26)$.

De fato, o estudo sobre CR encontra-se numa fase efervescente, e os resultados têm caminhado na direção oposta aos achados dos estudos realizados antes de Salmoni et al. (39). Em outras palavras, no conjunto de resultados atuais há forte tendência de relação de U invertido, ou seja, regimes de CR com freqüências e precisões máximas ou mínimas, em vez de facilitarem a aprendizagem, na realidade prejudicam-na.

Entretanto, apesar dessas novas descobertas sobre os efeitos de CR, é importante ressaltar que existe um problema de fundo que coloca sérias limitações mesmo aos achados nessa nova fase de investigações pós Salmoni et al. (39). Trata-se da concepção de aprendizagem motora subjacente a essas investigações que considera a aquisição de habilidades motoras um processo finito que se encerra com a automatização do movimento. 
Na realidade, a aquisição de habilidades motoras é por natureza um processo dinâmico e complexo. Todavia, as teorias correntes de aprendizagem motora explicam apenas uma parte desse processo, qual seja, a estabilização da performance que se caracteriza como um processo homeostático (equilíbrio) alcançado via feedback negativo $(1,41)$. Processos baseados em feedback negativo, ou mecanismo de neutralização do desvio (34), são capazes de manter a estrutura ou ordem, mas são incapazes de conduzir a uma nova estrutura, visto que para tanto, é necessário desestabilização. A automatização, vista como a fase final do processo de aprendizagem motora pelas teorias correntes, é um exemplo típico de estabilização.

Diante dessa situação, Choshi $(18,19,20)$, Choshi e Tani $(21)$, Tani $(48,49)$ e Tani, Bastos, Castro, Jesus, Sacay e Passos (50) têm proposto um modelo de não-equilíbrio em aprendizagem motora em que dois processos fundamentais são considerados: estabilização e adaptação. O primeiro é aquele em que se busca, como a própria palavra indica, a estabilidade funcional que resulta na padronização espacial e temporal do movimento (formação de estrutura). Movimentos inicialmente inconsistentes vão sendo gradativamente refinados até se alcançar movimentos padronizados e precisos. Nesse processo, o elemento fundamental é o feedback negativo. O segundo é aquele em que se procura adaptações às novas situações ou tarefas motoras (perturbação), mediante a aplicação das habilidades já adquiridas. Nesse processo, exigem-se modificações na estrutura da habilidade já adquirida, e uma posterior reorganização dessa estrutura num nível superior de complexidade. Existem perturbações para as quais a adaptação se faz pela flexibilidade inerente à estrutura adquirida, ou seja, pela mudança de parâmetros do movimento. Contudo, existem perturbações de tal envergadura que por mais que haja disponibilidade na estrutura não há condições de adaptar-se. Nesse caso, exige-se uma reorganização da própria estrutura que, quando concluída, resulta numa mudança qualitativa do sistema (48).

Em resumo, na aprendizagem motora é importante não só a estabilização funcional, mas também a capacidade de aplicação das habilidades adquiridas às novas situações ou tarefas motoras. Nessa visão, a aquisição de habilidades motoras melhor caracteriza um processo cíclico e dinâmico de instabilidade-estabilidade-instabilidade, resultando em crescente complexidade. E dentro dessa perspectiva, fatores relacionados à incerteza, instabilidade e desordem podem não desempenhar papel negativo no decorrer do processo. Recentes proposições dentro do paradigma sistêmico têm enfatizado que a formação de novas estruturas pressupõe instabilidade ou quebra de estabilidade (49 e 50).

Dessa forma, o CR como redutor de incerteza para o aprendiz pode atuar como um fator importante, porém não essencial. Em outras palavras, pode ser fornecido para guiá-lo ao alcance da meta, mas com certa margem de tolerância. O fornecimento de CR pode inibir o envolvimento ativo do aprendiz no processo de aprendizagem para desenvolver a capacidade de detecção e correção de erros. Isso posto, o presente estudo pretende investigar a variável CR como fator de incerteza na aquisição de uma habilidade motora. Especificamente, o objetivo do presente estudo é investigar o efeito de CR no processo adaptativo na aquisição de uma habilidade motora de controle de força manual, considerando as variáveis, precisão, freqüência e localização temporal de CR.

\section{EXPERIMENTO 1: FREQÜÊNCIA DE CR}

1.1. Tarefa motora, equipamento e medida de desempenho A tarefa motora envolveu o controle do movimento de pressionar um dinamômetro manual (Takei \& Co.) com o propósito de aplicar uma porcentagem da sua força máxima. A cada movimento executado, o mostrador eletrônico do dinamômetro indicava um número correspondente à quantidade de força máxima (expressa em kgf) aplicada pelos dedos na preensão da manopla. A precisão de medida foi de $0,5 \mathrm{kgf}$. Como medida, utilizou-se o erro absoluto que é a diferença, em módulo, entre o valor desejado e o manifesto.

\subsection{Sujeitos}

Oitenta alunos do Curso de Graduação da Escola de Educação Física e Esporte da Universidade de São Paulo participaram voluntariamente como sujeitos. As idades variaram entre 18 e 29 anos (média de $23,4 \pm 4,1$ anos). Todos eram inexperientes com experimentos em aprendizagem motora e conseqüentemente com a tarefa proposta. 


\subsection{Delineamento experimental}

Os sujeitos foram divididos aleatoriamente em quatro grupos (G100, G66, G33, G20), cada qual com vinte elementos. A cada grupo foi fornecido CR com determinada freqüência. Na fase de estabilização, a freqüência absoluta foi de dezoito (18) informações de CR para todos os grupos, com freqüência relativa de $100,66,33$ e $20 \%$, respectivamente, para G100, G66, G33 e G20, cabendo para estes mesmos grupos, portanto, um total de 20, 29, 58 e 96 tentativas a executar. Para todos os grupos, a informação de CR contemplou magnitude e direção do erro. $\mathrm{Na}$ fase de adaptação, todos os sujeitos executaram 10 tentativas sem o fornecimento de CR.

\subsection{Procedimentos}

Os sujeitos foram orientados sobre o manuseio do aparelho, com especificações sobre a maneira de pressioná-lo e a postura necessária para tal procedimento. Inicialmente foi medida a força de preensão manual máxima em duas tentativas e, com base no maior valor obtido, foi calculado o valor critério a ser alcançado (50\% na fase de estabilização e $30 \%$ na fase de adaptação). Respeitou-se um intervalo de descanso de 20 segundos entre as tentativas. Após a execução de cada tentativa, o aparelho foi entregue ao experimentador para que informações adicionais (cinestésicas) não pudessem interferir na execução da próxima tentativa. Não foi permitido aos sujeitos olhar para o resultado no aparelho, ou seja, não lhes foi possível obter feedback por meios próprios.

\subsection{Resultados}

As dezoito tentativas pós-CR de estabilização e as nove tentativas de adaptação foram organizadas, respectivamente, em seis blocos e três blocos (três tentativas cada bloco). A análise considerou a soma dos erros em cada bloco. As curvas de desempenho dos grupos podem ser visualizadas na Figura 1.

A realização de uma estatística inferencial foi necessária para identificar diferenças inter e intra-grupos. Os pressupostos de normalidade e homogeneidade de variâncias permitiram a realização de duas análises de variância com medidas repetidas no segundo fator "grupo x bloco" 4x6 (estabilização) e 4x3 (adaptação). O teste post-hoc de Tukey identificou a localização das diferenças significativas. Na fase de estabilização, para o fator grupo houve diferença sig- nificativa $\left(\mathrm{F}_{3,76}=10,26 ; \mathrm{p}<0,001\right)$ : o $\mathrm{G} 66$ errou mais que o G100, e este mais que o G33 e G20. Já no fator bloco, os valores permitem inferir que os sujeitos, sem distinção de grupo, aprenderam a tarefa $\left(\mathrm{F}_{5,380}=24,31 ; \mathrm{p}<0,001\right)$. A interação grupo $\mathrm{x}$ bloco apresentou valor de prova maior que 0,05 .

$\mathrm{Na}$ fase de adaptação, não foram detectadas diferenças significativas na interação e no fator bloco.

Todavia, a análise do fator grupo $\left(\mathrm{F}_{3,76}=4,16\right.$; $\mathrm{p}<0,01)$ detectou que o $\mathrm{G} 66$ obteve pior performance em relação aos demais.

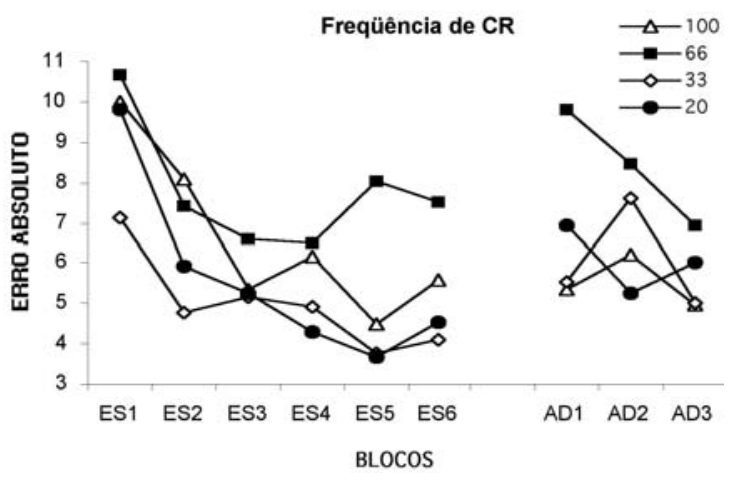

Figura 1: Médias dos erros absolutos dos grupos ao longo das fases do experimento.

EXPERIMENTO 2: PRECISÃO DE CR

2.1. Tarefa motora, equipamento e medida de desempenho e procedimentos

Idem ao experimento 1.

\subsection{Sujeitos}

Oitenta alunos (diferentes daqueles do experimento 1) do Curso de Graduação da Escola de Educação Física e Esporte da Universidade de São Paulo participaram voluntariamente como sujeitos. As idades variaram entre 17 e 27 anos (média de 23,1 14,5 anos). Todos eram inexperientes com experimentos em aprendizagem motora e conseqüentemente com a tarefa proposta.

\subsection{Delineamento experimental}

A manipulação experimental ocorreu com relação à direção e magnitude do erro. Os sujeitos foram divididos aleatoriamente em quatro grupos de vinte sujeitos: 1) controle - sem CR (Gsem); 2) apenas CR 
sobre direção do erro (Gdir); 3) apenas CR sobre magnitude do erro (Gmag); e 4) CR sobre magnitude e direção do erro (Gmag+dir). Todos os sujeitos executaram 20 tentativas para estabilização da performance. A fase de adaptação constou de 10 tentativas sem o fornecimento de CR.

\subsection{Resultados}

Os dados foram analisados em blocos de cinco tentativas em ambas as fases. A análise considerou a soma dos erros em cada bloco. Pode-se visualizar os resultados descritivos da análise na Figura 2. A identificação de diferenças inter e intra-grupos somente foi possível por meio de uma estatística inferencial (análise de variância com medidas repetidas no segundo fator e posterior teste post-hoc de Tukey), a qual foi executada após a confirmação de suas assunções básicas (normalidade e homogeneidade de variâncias).

Na fase de estabilização, a análise de variância 4 (grupo) x 4 (bloco) identificou diferenças para a interação dos fatores $\left(\mathrm{F}_{9,228}=3,13 ; \mathrm{p}<0,01\right)$. Nos três últimos blocos, o Gsem obteve desempenho inferior aos demais grupos. Detectou-se também diferença intra-grupo no Gdir, no Gmag e no Gmag + dir do primeiro bloco para os demais, indicando ocorrência de aprendizagem.

$\mathrm{Na}$ fase de adaptação, não foram detectadas diferenças significativas na interação, mas sim no fator grupo $\left(\mathrm{F}_{3,76}=14,32 ; \mathrm{p}<0,001\right)$. Visualmente, considerando os valores da média e do erro padrão, podese perceber que há nítida desvantagem do GsemCR para o Gmag; este, por sua vez, também apresentou desvantagem em relação ao Gdir e ao Gmag+dir.

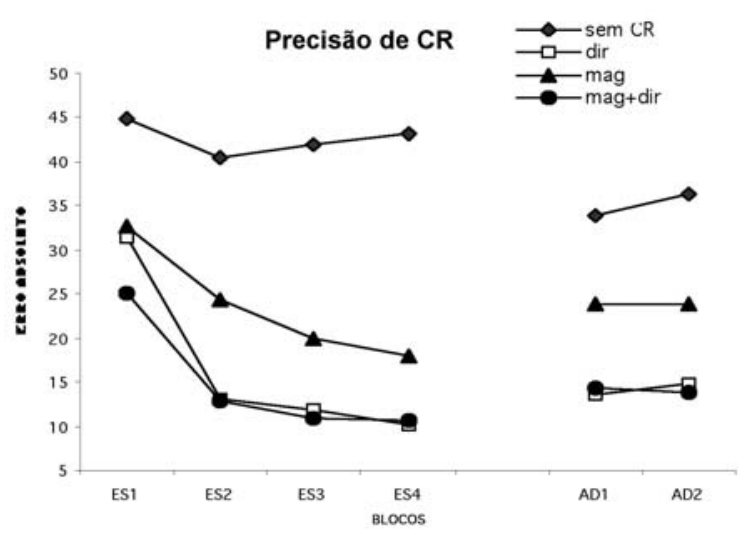

Figura 2: Média dos erros absolutos dos grupos ao longo das fases do experimento.

\section{EXPERIMENTO 3: ATIVIDADE INTERPOLADA DURANTE O INTERVALO PÓS-CR}

\subsection{Tarefa motora, equipamento e medida} de desempenho e procedimentos

Idem ao experimento 1 .

\subsection{Sujeitos}

Trinta e quatro alunas do Curso de Graduação da Escola de Educação Física e Esporte da Universidade de São Paulo tomaram parte voluntariamente como sujeitos da pesquisa. As idades variaram entre $18 \mathrm{e}$ 31 anos (média de 24,6 $\pm 4,4$ anos). Todas eram inexperientes com experimentos em aprendizagem motora e conseqüentemente com a tarefa empregada.

\subsection{Delineamento experimental}

Os sujeitos foram divididos aleatoriamente em dois grupos: 1) com a realização de uma tarefa de tapping durante o intervalo pós-CR (Gcom) e 2) sem realização de tarefa durante o intervalo pós-CR (Gsem). Os membros do Gcom executaram a tarefa de tapping com a mão não-dominante da forma mais rápida possível. Todos os sujeitos realizaram 24 tentativas na fase de estabilização, na qual, após cada tentativa, os sujeitos receberam CR sobre magnitude e direção do erro. O intervalo pré-CR durou cinco segundos e o intervalo pós-CR durou 15 segundos. Na fase de adaptação, todos os sujeitos executaram 6 tentativas sem o fornecimento de CR.

\subsection{Resultados}

Em ambas as fases do experimento, os dados foram organizados em blocos de 3 tentativas, considerando a soma no bloco para a análise estatística. Os valores descritivos podem ser visualizados na Figura 3.

Depois de confirmadas a normalidade da distribuição e a homogeneidade de variâncias, duas análises de variância com medidas repetidas no segundo fator grupo $\mathrm{x}$ bloco foram utilizadas como técnicas paramétricas para identificação de diferenças inter e intra-grupos, uma 2 × 8 na estabilização e uma 2 × 2 na adaptação. As diferenças foram localizadas pelo teste post-hoc de Tukey.

$\mathrm{Na}$ fase de estabilização, detectou-se diferença significativa apenas para o fator bloco $\left(\mathrm{F}_{7,224}=30,21\right.$ $\mathrm{p}<0,001)$. Esses valores permitem inferir que os sujeitos, sem distinção de grupo, aprenderam a tare- 
fa. Não se identificaram diferenças significativas na fase de adaptação. Considerando os valores descritivos representados na Figura 3, o Gcom foi melhor que o Gsem no primeiro bloco, situação que se inverteu no segundo bloco.

\section{Atividade no intervalo pós-CR}

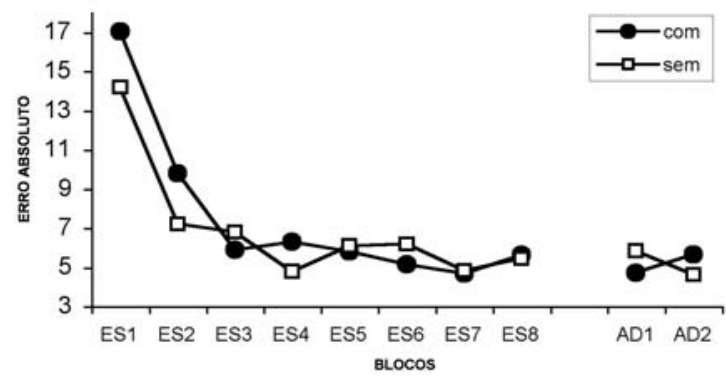

Figura 3: Média dos erros absolutos dos grupos ao longo das fases do experimento.

\section{DISCUSSÃO E CONCLUSÕES}

O objetivo do presente estudo foi investigar o efeito do CR no processo adaptativo em aprendizagem motora, considerando precisão, freqüência e localização temporal. Esperava-se que regimes de CR menos freqüentes, menos precisos e com atividade no intervalo pós-CR (associados a fatores de instabilidade, incerteza e desordem) não prejudicassem o processo adaptativo em aprendizagem motora. $\mathrm{O}$ conjunto dos resultados apontou nessa direção. Inicialmente deve-se destacar que todos os regimes que receberam CR durante a fase de estabilização evoluíram significativamente do início para o final, ou seja, a aprendizagem da tarefa esteve intimamente ligada ao fornecimento de $\mathrm{CR}$, variável que se mostra crucial durante o processo. De maneira geral, os resultados corroboram as hipóteses gerais, uma vez que regimes de CR com freqüências menores, sem muita precisão e com atividades interpoladas durante o intervalo pós-CR não prejudicaram o processo adaptativo. Esses achados alinham-se a correntes recentes de estudos sobre $\mathrm{CR}$, baseadas no processamento de informações $(12,16,53,54,55,56)$.

No que concerne ao intervalo pós-CR, a literatura aponta que atividade interpolada de caráter cognitivo prejudica a aprendizagem da tarefa principal $(6,47)$; já uma atividade interpolada de natureza motora pode otimizar a aprendizagem da tarefa principal (32). Como no intervalo pós-CR o aprendiz se envolve em uma tarefa cognitiva de comparação entre suas próprias informações (feedback intrínseco) e a informação externa (CR), é de se esperar que se a tarefa secundária for de natureza cognitiva, haverá alguma interferência negativa para a aprendizagem. A explicação para a otimização da aprendizagem com interpolação de tarefa motora durante o intervalo pós-CR está sustentada na idéia de transferência positiva de solução de problemas da situação enfrentada na fase de aquisição e da situação em que o aprendiz é exposto nos testes de aprendizagem (28). No presente estudo, o fator de instabilidade engendrado pela atividade interpolada de tapping executada na fase de estabilização não prejudicou o desempenho na fase de adaptação.

A partir da publicação do artigo seminal de Salmoni et al. (39), começou-se a questionar a idéia de que melhor seria a aprendizagem quanto mais preciso fosse o CR, ou seja, quando a informação era fornecida sobre magnitude e direção do erro. Desde então, o conjunto de estudos não permite extrair conclusões sólidas sobre qual regime de precisão de CR otimiza a aprendizagem $(5,15,33,36,38,40)$. A considerar os resultados do presente trabalho, é possível advogar regimes de CR gerais - haja vista que o grupo que recebeu $\mathrm{CR}$ apenas sobre direção do erro (geral) obteve controle adaptativo igual ao grupo que recebeu CR sobre magnitude e direção do erro (específico). Desse modo, não se faz necessário reduzir excessivamente a incerteza por intermédio de regimes de CR com muita precisão.

O artigo de Salmoni et al. (39) também influenciou de forma determinante a noção estabelecida pelas teorias clássicas de aprendizagem motora $(1,41)$ de que quanto mais CR, melhor a aprendizagem. Embora não de forma explícita, essas teorias apregoavam regimes de $\mathrm{CR}$ com freqüências altas. Entretanto, o que se verificou após a proposta de Salmoni et al. (39) foi um número substancial de trabalhos fornecendo suporte a regimes de CR reduzidos como mais eficazes para a aprendizagem de habilidades motoras $(14,25,53,54,55,56)$. A maior incerteza provocada por regimes de CR com 
freqüências menores também não prejudicou o controle adaptativo na aprendizagem da tarefa empregada no presente estudo.

Regimes reduzidos de CR têm encontrado suporte em duas hipóteses explicativas: a hipótese da instabilidade - maladaptive short-term corrections (42) e a hipótese da orientação - guidance hypothesis $(39,42)$. De acordo com a primeira, regimes de CRs freqüentes - uma vez que em toda tentativa há correção não proporcionam oportunidade para a aquisição de consistência, importante nos testes de aprendizagem (esse é um exemplo claro de que o estudo do CR tem-se dado sob uma perspectiva de equilíbrio, no caso porque enfatiza apenas a consistência). A segunda hipótese postula que CR freqüente causa dependência de fornecimento, o que prejudica o desenvolvimento da capacidade de detectar e corrigir erros, em virtude da inibição do processamento da informação intrínseca.

A hipótese explicativa para os resultados do presente trabalho alinha-se às anteriores no que diz respeito à assunção de que regimes de CR freqüentes, precisos e com atividades interpoladas são prejudiciais à aquisição de habilidades motoras. No entanto, a hipótese de explicação lançada aqui diferencia-se das hipóteses da instabilidade e da orientação quanto à maneira de abordar o processo (no caso, como de não equilíbrio) e também quanto ao argumento. No modelo de processo adaptativo, propõe-se que o programa de ação é organizado hierarquicamente, ou seja, uma macroestrutura orientada à ordem (consistência) e uma microestrutura orientada à desordem (variabilidade). Regimes de CR freqüentes e precisos estimulariam: a) correções (diminuição do erro) nos detalhes de movimento que implicariam redução da variabilidade na microestrutura do programa de ação, resultando na formação de um programa sem a necessária flexibilidade para adaptação - isso implicaria em rigidez na parametrização do movimento; b) aumento da dependência a informações extrínsecas que resultaria na não utilização e aprimoramento do mecanismo de detecção e correção de erros mediante exploração do feedback intrínseco e com isso a autonomia do sistema não estaria sendo devidamente desenvolvida; e c) o CR freqüente e preciso implicaria a inibição excessiva da incerteza dentro do sistema, incerteza essa que é necessária para garantir o fluxo de energia livre ou informação, visto que quando o sistema está à busca de estabilidade (diminuição do fluxo de energia livre ou informação) necessita, ao mesmo tempo, desenvolver capacidade que o permita adaptar-se às novas situações ou tarefas. Conclui-se, portanto, que a redução da freqüência e precisão do $\mathrm{CR}$, assim como as atividades interpoladas pós-CR, podem constituir-se fontes de instabilidade não prejudiciais no processo adaptativo em aprendizagem motora.

\section{CORRESPONDÊNCIA}

\section{Go Tani}

Escola de Educação Física e Esporte

Universidade de São Paulo

Av. Prof. Mello Moraes, 65 - São Paulo/SP

05508-900 Brasil

gotani@usp.br 


\section{REFERÊNCIAS BIBLIOGRÁFICAS}

1. Adams, J.A. (1971). A closed-loop theory of motor learning. Journal of Motor Behavior 3:111-150.

2. Adams, J.A. (1987). Historical review and appraisal of research on the learning, retention, and transfer of human motor skills. Psychological Bulletin 101:41-74.

3. Anderson, D.I.; Magill, R.A.; Sekiya, H. (2001). A reconsideration of the trials-delay of knowledge of results paradigm in motor skill learning. Research Quarterly for Exercise and Sport 65: 286-290.

4. Bartlett, F.C. (1948). The measurement of human skill. Occupational Psycology 22: 31-38.

5. Bennett, D.M.; Simmons, R.W. (1984). Effects of the precision of knowledge of results on acquisition and retention of a simple motor skill. Perceptual and Motor Skills 58: 785786.

6. Benedetti, C.; McCullagh, P. (1987). Post-knowledge of results delay: effects of interpolated activity on learning and performance. Research Quarterly for Exercise and Sport 58: 375-381.

7. Bilodeau, E.A.; Bilodeau, I.M. (1958). Variable frequency of knowledge of results and the learning of a simple skill. Journal of Experimental Psychology 55: 379-383.

8. Boren, J.J. (1961). Resistance to extinction as a function of the fixed ratio. Journal of Experimental Psychology 61: 304308.

9. Boulter, L.R. (1964). Evaluation of mechanisms in delay of knowledge of results. Canadian Journal of Psychology 18: 281-291.

10. Buekers, M.J.; Magill, R.A.; Hall, K.G. (1992). The effect of erroneous knowledge of results on skill acquisition when augmented information is redundant. Quarterly Journal of Experimental Psychology 45A: 105-117.

11. Buekers, M.J.; Magill, R.A.; Sneyers, K.M. (1994). Resolving a conflict between sensory feedback and knowledge of results, while learning a motor skill. Journal of Motor Behavior 26: 27-35.

12. Castro, I.J. (1988). Efeitos da freqüência relativa do feedback extrínseco na aprendizagem de uma habilidade motora discreta simples. Dissertação (Mestrado) - Escola de Educação Física e Esporte, Universidade de São Paulo. São Paulo.

13. Chen, D.D. (2002). Catching the learner doing right versus doing wrong: effects of bandwidth knowledge of results orientations and tolerance range sizes. Journal of Human Movement Studies 42: 141-154.

14. Chiviacowsky, S. (2000). Efeitos da freqüência do conhecimento de resultados controlada pelo experimentador e autocontrolada pelos sujeitos na aprendizagem de tarefas motoras com diferentes complexidades. Tese (Doutorado) Faculdade de Motricidade Humana, Universidade Técnica de Lisboa. Lisboa.

15. Chiviacowsky, S.; Godinho, M. (1997). Aprendizagem de habilidades motoras em crianças: algumas diferenças na capacidade de processar informações. Boletim da Sociedade Portuguesa de Educação Física 15/16: 39-47.

16. Chiviacowsky, S.; Tani, G. (1993). Efeitos da freqüência de conhecimento de resultados na aprendizagem de uma habilidade motora em crianças. Revista Paulista de Educação Física 7: 45-57.

17. Chiviacowsky, S.; Wulf, G. (2002). Self-controlled feed- back: does it enhance learning because performers get feedback when they need it? Research Quarterly for Exercise and Sport 73: 408-415.

18. Choshi, K. (1978). The organization of perceptual-motor behavior. In H. Hagiwama \& K. Choshi (Eds.), The organization of perceptual-motor bahavior. Tokyo: Fumaido (in Japanese).

19. Choshi, K. (1981). The significance of error response in adaptive systems. Sport Psychology Research 7: 60-64 (in Japanese).

20. Choshi, K. (1982). An analytical study of the adaptive process in motor learning. Memoirs of the Faculty of Integrated Arts and Sciences III. Hiroshima University, 6: 75-82 (in Japanese).

21. Choshi, K.; Tani, G. (1983). Stable system and adaptive system in motor learning. In Japanese Association of Biomechanics (Ed.) The science of movement $V$. Tokyo: Kyorin (in Japanese).

22. Dyal, J. (1966). Effects of delay of knowledge of results and subject response bias on extinction of a simple motor skill. Journal of Experimental Psychology 71: 559-563.

23. Gable, C.D.; Shea, C.H.; Wright, D.L. (1991). Summary knowledge of results. Research Quarterly for Exercise and Sport 62: 285-292.

24. Goodwin, J.E.; Meeuwsen, H.J. (1995). Using bandwidth knowledge of results to alter relative frequencies during motor skill acquisition. Research Quarterly for Exercise and Sport, 66: 99-104.

25. Guadagnoli, M.A.; Kohl, R.M. (2001). Knowledge of results for motor learning: relationship between error estimation and knowledge of results frequency. Journal of Motor Behavior 33: 217-224.

26. Janelle, C.M.; Barba, D.A.; Frehlich, S.G.; Tennant, L.K.; Cauraugh, H. (1997). Maximizing performance feedback effectiveness through videotape replay and a self-controlled learning environment. Research Quarterly for Exercise and Sport 68: 269-279.

27. Janelle, C.M.; Kim, J.; Singer, R.N. (1995). Subject-controlled performance feedback and learning of a closed motor skill. Perceptual and Motor Skills 81: 627-634.

28. Lee, T.D. (1988). Testing for motor learning: a focus on transfer-appropriate-processing. In O.G. Meijer and K. Roth (Eds.) Complex motor behaviour: "the" motor-action controversy. Amsterdam: Elsevier Science Publishers, 210-215.

29. Lee, T.D.; Magill, R.A. (1985). Can forgetting facilitate skill acquisition? In D. Goodman, R.B. Wilberg \& I.M. Franks (Eds.) Differing perspectives in motor learning, memory and control. Amsterdam: Elsevier Science Publishers, 3-22.

30. Lorge, I.; Thorndike, E.L. (1935). The influence of delay in the after effect of a connection. Journal of Experimental Psychology 18: 186-194.

31. Lustosa de Oliveira, D. (2002). Freqüência relativa de conhecimentos de resultados (CR) e complexidade da tarefa na aprendizagem de habilidades motoras. Dissertação (Mestrado) - Escola de Educação Física e Esporte, Universidade de São Paulo, São Paulo.

32. Magill, R.A. (1988). Activity during the post-knowledge of results interval can benefit motor skill learning. In O.G. Meijer \& K. Roth (Eds.) Complex motor behavior: "the" motor-action controversy. Amsterdam: Elsevier Science Publishers, 231-246.

33. Magill, R.A.; Wood, C.A. (1986). Knowledge of results 
precision as a learning variable in motor skill acquisition. Research Quarterly for Exercise and Sport 57: 170-173.

34. Maruyama, M. (1963). The second cybernetics: deviationamplifying mutual causal processes. American Scientist 51: 164-179.

35. McNevin, N.; Magill, R.A.; Buekers, M.J. (1994). The effects of erroneous knowledge of results on transfer of anticipation timing. Research Quarterly for Exercise and Sport 64: 324-329.

36. Newell, K.M.; Kennedy, J.A. (1978). Knowledge of results and children's motor learning. Developmental Psychology 14: 531-536.

37. Newell, K.M.; Quinn, J.T.; Sparrow, W.A.; Walter, C.B. (1983). Kinematic information feedback for learning a rapid arm movement. Human Movement Science 2: 255-269.

38. Rogers Jr., C.A. (1974). Feedback precision and post-feedback interval duration. Journal of Experimental Psychology 102: 604-608.

39. Knowledge of results and motor learning: a review and critical reappraisal. Psychological Bulletin 95: 355-386.

40. Scaringe, J.G.; Chen, D.P.; Ross, D. (2002). The effects of augmented sensory feedback precision on the acquisition and retention of a simulated chiropractic task. Journal of Manipulative and Physiological Therapeutics 25: 345-41.

41. Schmidt, R.A. (1975). A schema theory of discrete motor skill learning. Psychological Review 82: 225-260.

42. Schmidt, R.A. (1991). Motor learning and performance: from principles to practice. Champaign, Illinois: Human Kinetics.

43. Schmidt, R.A.; Young, D.E.; Swinnen, S.; Shapiro, D.C. (1989). Summary knowledge of results for skill acquisition: support for the guidance hypothesis. Journal of Experimental Psychology: Learning, Memory and Cognition 15: 352-359.

44. Sherwood, D.E. (1988). Effect of bandwidth knowledge of results on movement consistency. Perceptual and Motor Skills 66: 535-542.

45. Skinner, B.F. (1953). Science and human behavior. New York: Macmillan.

46. Smith, P.J.; Taylor, S.J.; Withers, K. (1997). Applying bandwidth feedback scheduling to a golf shot. Research Quarterly for Exercise and Sport 68: 215-221.

47. Swinnen, S.P. (1990). Interpolated activities during the knowledge of results delay and post-knowledge of results interval: effects of performance and learning. Journal of Experimental Psychology: Learning, Memory, and Cognition 16: 692-705.

48. Tani, G. (1982). Adaptive process in perceptual-motor skill learning. Unpublished Doctoral Dissertation. Hiroshima. Faculty of Education, Hiroshima University (in Japanese).

49. Tani, G. (1989). Variabilidade de resposta e processo adaptativo em aprendizagem motora. Tese (Livre-Docência) Escola de Educação Física da Universidade de São Paulo, São Paulo.

50. Tani, G.; Bastos, F.C.; Castro, I.J.; Jesus, J.F.; Sacay, R.C.; Passos, S.C.E. (1992). Variabilidade de resposta e processo adaptativo em aprendizagem motora. Revista Paulista de Educação Física 6: 16-25.

51. Thorndike, E.L. (1927). The law of effect. American Journal of Psychology v.39: 212-222.

52. Trowbridge, M.H.; Cason, H. (1932). An experimental study of Thorndike's theory of learning. Journal of General Psychology v.7: 245-258.
53. Winstein, C.J.; Schmidt, R.A. (1990). Reduced frequency of knowledge of results enhances motor skill learning. Journal of Experimental Psychology: Learning, Memory and Cognition v.16: 677-691.

54. Wulf, G. (1992). Reducing knowledge of results can produce context effects in movements of the same class. Journal of Human Movement Studies v.22: 71-84.

55. Wulf, G. (1992). The learning of generalized motor programs and motor schemata: effects of KR frequency and contextual interference. Journal of Human Movement Studies v.23: $53-76$

56. Wulf, G.; Lee, T.D.; Schmidt, R.A. (1994). Reducing knowledge of results about relative versus absolute timing: differential effects on learning. Journal of Motor Behavior v.26: 362-369.

57. Wulf, G.; Schmidt, R.A. (1996). Average KR degrades parameter learning. Journal of Motor Behavior v.28: 371-381.

58. Wulf, G.; Schmidt, R.A. (1994). Feedback-induced variability and the learning of generalized motor programs. Journal of Motor Behavior v.26: 348-361.

59. Yao, W.X. (2003). Average-KR schedule benefits generalized motor program learning. Perceptual and Motor Skills v.97: 185-191. 